\title{
PHOTOELASTICITY BASED DYNAMIC TACTILE SENSOR
}

\author{
Venketesh N Dubey \\ Bournemouth University \\ School of Design, Engineering \& Computing \\ Talbot Campus, Fern Barrow \\ Poole, BH12 5BB, UK \\ Tel. +44 1202965986 \\ Fax. +44 1202965314 \\ Email:vdubey@bmth.ac.uk
}

\author{
Richard M Crowder \\ University of Southampton \\ Intelligence, Agents, Multimedia Group \\ School of Electronics and Computer Science \\ Southampton, SO17 1BJ, UK \\ Tel. +442380593441 \\ Fax. +442380592865 \\ Email: rmc@ecs.soton.ac.uk
}

\begin{abstract}
The paper presents design, construction and testing of a photoelasticity based dynamic sensor which is capable of detecting slip as well as providing normal force information. Starting with investigations into mechanism of slip, an approximate model of the sensor has been developed. This model explains the design improvements necessary to provide continuous signal during slip. The theoretical model also helps identify various sensor parameters to characterize the sensor. The developed sensor has been compared with other existing sensors and the experimental results from the sensor have been discussed for the type of signal the sensor provides. The sensor is also calibrated for normal force. The sensor is novel in the sense that it offers dynamic slip signal as well as the normal force information from a single contact location, it provides continuous signal during slip, and it has small size which can be easily incorporated into robotic fingers. The sensor has an edge over other existing sensors that its design is simple yet it provides strong signals which are largely unaffected by external disturbances.
\end{abstract}

Keywords: Slip sensor, Photoelasticity, Tactile sensor, Dynamic sensing, Shear sensing.

\section{INTRODUCTION}

Optimal object grasp by robotic end effectors requires that the grasp force be maintained just above the minimum. This is to minimize the power consumption and to avoid excessive gripping force without causing damage to the object and without slipping it. These conditions of gentle grasp can be achieved if the object is grasped with a small force and the gripping force is increased when the object starts to slip. This requires detection of object slip. Also in some cases of object manipulation, detection of slip is necessary to cause sliding motions of fingers over the object surface [1].

The need for slip detection of object has long been realized for prosthetic applications [2] and has been considered useful in object manipulation [3]. However, although the idea of using slip has been emphasized for grasping and manipulation, it has also been reported that detection of slip is 'difficult to achieve' and often shadowed by external disturbances $[4,5]$.

Many different techniques have been employed to detect slip, however, none have compared or even remotely close to the sensitivity and robustness of human slip sensing capabilities. For robotic applications this has been achieved by a number of different techniques such as interpretation of tactile-array data, interpretation of touch-sensor data or by employing dedicated slip sensors [6]. A basic method of slip sensing was based on the 'lift and try' technique, where a change in motor current was monitored to assess the quality of 
the grip. This technique had obvious difficulties of differentiating gripping force from interaction force and had to start every time with a very small gripping force to reach to a large force when the part did not slip.

Some other approaches of technological importance in slip sensing [7] include a sapphire needle protruding from a sensor surface and touching the slipping object to generate vibrations, which in turn produces voltage from a piezoelectric crystal or from a permanent magnet and coils in a damping medium. Optical interrupt-type of slip sensors have been designed with slotted roller [8] which interrupts an optical path passing across it giving an indication of slip. Though this sensor gives a good indication of the speed and direction of slip the obvious disadvantages are poor slip resolution and the jamming of the roller. Additionally, if the sensor is used for grasping, the rolling contact at the object surface will tend to slip the object and stable grasp cannot be maintained.

In order to emulate the slip sensing capability of the natural hand, a number of pressure sensing sites have been created over the sensing surface. As the object slips, a change in pressure detected by sensors gives an indication of slip, however, it is difficult to differentiate slip of object with applied force changes to the sensing sites.

Most of the techniques used for slip sensing rely on one of the above methods, however, the measurand in various approaches are different. Kyberd and Chappell [9] have developed a slip sensor, based on forced oscillations which detects the sound produced by vibrations using a microphone. Kvasnik et al. [10] have used photoelastic material to detect slip. The sensor was reported to detect start and termination of slip. Eghtedari and Morgan [11] have used photoelastic material and a solid-state camera with a detailed processing to infer the object slip.

With the surge of VLSI chip fabrication technology many slip sensors are designed as sensing arrays. A slip sensor using a piezoelectric bimorph element has been developed by Nishihara et al. [12]. The sensor is sensitive to dynamic stress which detects the instant of first slip as well as contact and release of an object. However, the sensor is easily influenced by vibrations, for which vibration absorbers are used. Holweg et al. [13] have used rubber based tactile matrix sensor to detect slip, which rely on the elasticity of the rubber. Many recent developments in slip sensing employ the sensing array technology [14-18]. Although the research on slip sensing has emphasized the need for detection of incipient slippage for stable grasping and dexterous manipulation [19-21], such localized slip detection are often compounded by external disturbances.

The sensor described in this paper is based on the photoelastic principles and is capable of detecting slip as well as the normal force. It produces continuous slip signal and the signal is so strong that it does not require any amplification, leaving it largely immune to the external disturbances. Experiments conducted with this sensor have been reported.

\section{MECHANISM OF SLIP}

In the context of grasping and manipulation, slip may be regarded as the relative movement of one object surface over another when they are in contact. The relative motion may be of any form ranging from simple translational motion to a combination of translational and rotational motions. Mechanically, slip can be thought to be shearing of two object surfaces in contact, in all possible forms of motions. Visually it may be easier to detect relative movement between two slipping objects, however, it is difficult to exactly analyze various material and geometrical interactions in terms of physical behavior of the contacting surfaces during slip. Starting from the rest situation, the contact surfaces form a pressure distribution pattern which cannot be predicted accurately (this is due to the random irregularity of the contacting surfaces) and when the object slips, the pressure distribution is disturbed; the contact pressure however remains the same. How the pressure distribution changes, largely depends on the elastic nature of the objects, slip rate, surface roughness and the geometric boundary conditions. The change in pressure distribution, however, does not provide any detectable information (as opposed to the vision systems) about the object slip. In tactile sensing, associated side effects such as acoustic sound produced during slip, vibration of the slipping surface or the relative movement between contact surfaces can only be exploited to detect the object slip.

An illustration of an object slipping vertically downward between two fingers is shown in Fig. 1.

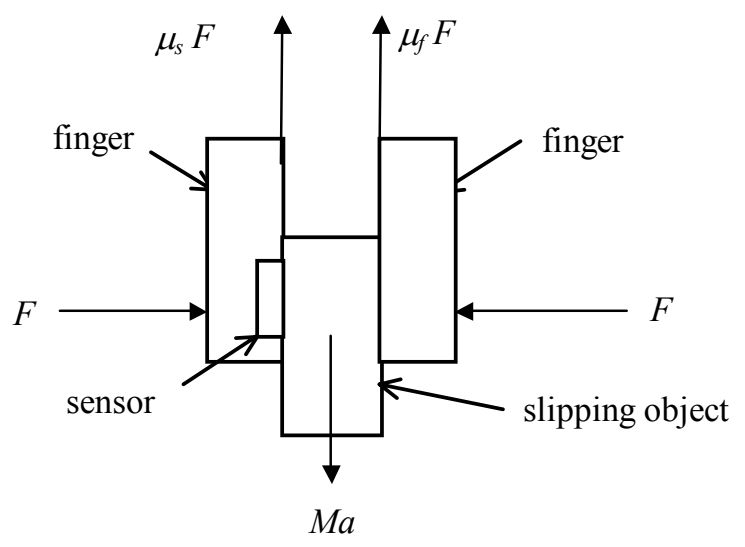

Figure 1. A slipping object with system of forces

The two fingers provide a force to hold the object stationary. It will slip if forces applied on the object including those due to gravity and acceleration are larger than the frictional forces, in which case,

$$
M a>F\left(\mu_{f}+\mu_{s}\right)
$$

Object will be at rest if,

$$
M a \leq F\left(\mu_{f}+\mu_{s}\right)
$$

where $\mu_{f}, \mu_{s}$ are the coefficients of friction between the finger/sensor and the object and $a$ is the vector sum of 
acceleration due to gravity together with that supplied by the inertia. To prevent the object slippage, the applied finger force $(F)$ needs to be increased.

\section{DESIGN OF THE SLIP SENSOR}

The layout of the developed sensor is shown in Fig. 2 where light emitted by a source is passed through the photoelastic layer (polyurethane PSM-4, Measurements Group Inc.) located between a polarizer and an analyzer. On entering the stressed transparent photoelastic material, the polarized light is split into two components which vibrate in two perpendicular planes of principal stresses. The velocity of propagation of each component of light ray differs from that in the unstressed material by an amount which depends on the magnitude of the principal stresses. Consequently, the two rays emerge from the material out of phase. The difference in phase depends on the difference between two principal stresses, the wavelength of light, thickness of the material in the direction of propagation of light, and the stress optical sensitivity of the material. The two rays emerging from the material are received by an analyzer, which only transmits the components of two rays in its plane of polarization causing a change in the intensity of light at the receiver.

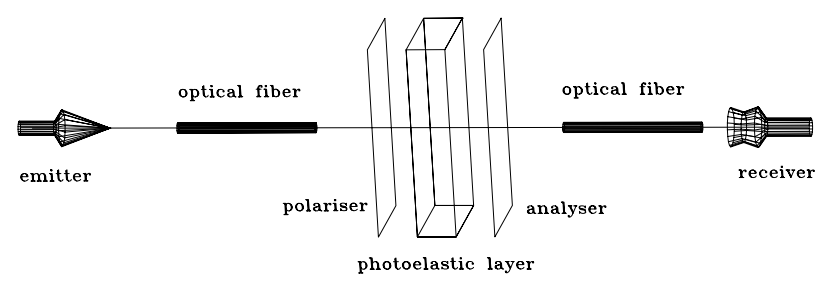

Figure 2. Layout of the sensor

Figure 3 shows the boundary conditions and dimensions of the photoelastic layer used in the sensor design. The layer has a fixed boundary forming a cantilever beam. The shaded portion forms the sensing area. The $l, b$ and $t$ are the length, breath and the thickness of the layer respectively.

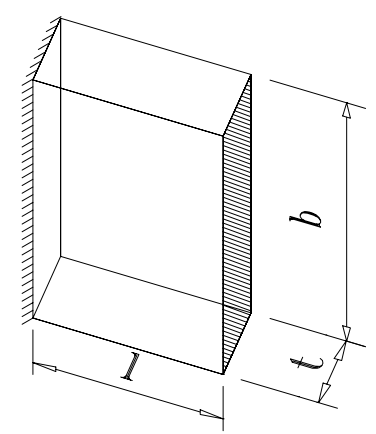

Figure 3. Sensor dimensions and boundary conditions

Under the action of the applied normal force and the shearing force for the case shown in Fig. 1, the free body diagram of the sensing material can be shown in Fig. 4:
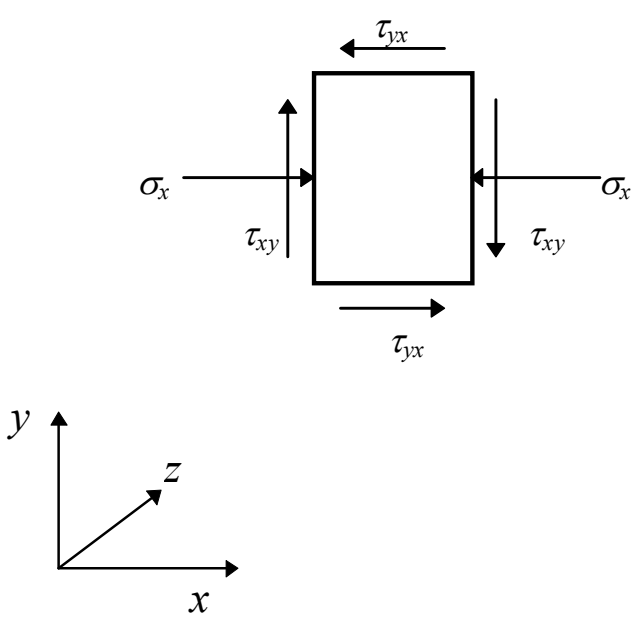

Figure 4. Modeling of the sensing medium

where $\sigma_{x}$ is normal stress along $x$-direction and $\tau_{x y}$ is the shear stress in $x y$-plane. The counter couple developed in the material is represented by the shear stresses $\tau_{y x}$ (opposite to $\tau_{x y}$ ). The normal and shear stresses can be expressed as,

$$
\begin{gathered}
\sigma_{x}=\frac{F}{A} \\
\tau_{x y}=\frac{\mu_{s} F}{A}
\end{gathered}
$$

$$
\text { and, } A=b \times t
$$

where $F$ is the applied fingertip force, $A$ is the surface area of the sensor and $\mu_{s}$ is the coefficient of friction between the sensor-object surfaces. This is the condition of plane stress in $x y$-plane with negligible $\sigma_{y}$ component. The magnitude of principal stresses $\sigma_{1}$ and $\sigma_{2}$ (with $\sigma_{y}=0$ ) is given by [22]:

$$
\begin{aligned}
& \sigma_{1}=\frac{\sigma_{x}}{2}+\frac{1}{2} \sqrt{\sigma_{x}^{2}+4 \tau_{x y}^{2}} \\
& \sigma_{2}=\frac{\sigma_{x}}{2}-\frac{1}{2} \sqrt{\sigma_{x}^{2}+4 \tau_{x y}^{2}}
\end{aligned}
$$

The orientation $(\alpha)$ of the principal stresses is defined as the angle between $x$-axis and the direction of the maximum principal stress $\left(\sigma_{l}\right)$ and is given by:

$$
\alpha=\frac{1}{2} \sin ^{-1}\left\{2 \tau_{x y} / \sqrt{\sigma_{x}^{2}+4 \tau_{x y}^{2}}\right\}
$$

The vector representation of the stresses is shown in Fig. 5. 


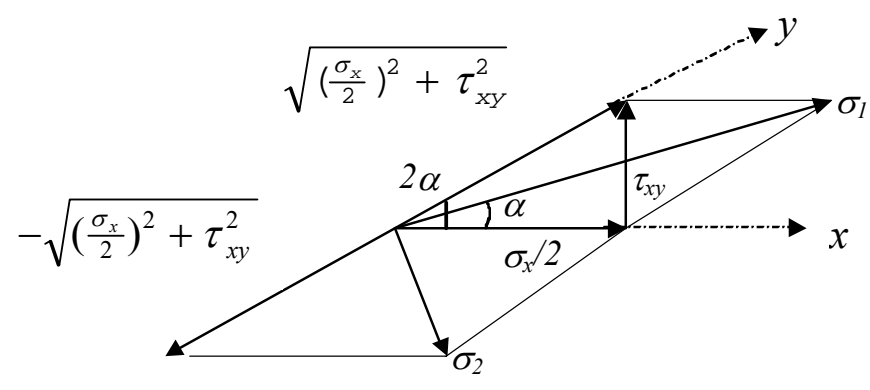

Figure 5. Principal stresses and principal planes

These relations are valid for semi-infinite solids but the sensor material considered here is a photoelastic layer which has finite dimensions so these relations are only an approximation for this application. Nevertheless, these relations give an idea of dependence of $\sigma_{1}, \sigma_{2}$ on the normal and shear stresses over it. The intensity of light $\left(I_{o}\right)$ emerging out of the analyzer is expressed as [23]:

$$
I_{o}=I_{i} \sin ^{2} 2 \theta \sin ^{2}\left\{\pi t\left(\sigma_{1}-\sigma_{2}\right) c / \lambda\right\}
$$

where $I_{i}$ is incident intensity of light, $\theta$ is angle between plane of principal stress $\left(\sigma_{l}\right)$ and the plane of polarization, $t$ is the thickness of the layer in the direction of light propagation, $\lambda$ is the wavelength of light used and $c$ is the stress optical coefficient (which is a known property of the material). The angle $\theta$ is shown in Fig. 6, where light enters the polarizer along the optical axis $\mathrm{O}$ (perpendicular to the plane of the paper). Light emerging from the polarizer has amplitude represented by the vector $\left(A_{p}\right)$ along the axis of polarizer. When this polarized light enter the stressed photoelastic material, it splits into two component vectors $\left(A_{p 1}\right.$ and $\left.A_{p 2}\right)$ along the planes of principal stresses. At analyzer (whose axis is shown to be perpendicular to the axis of the polarizer), the components of these two vectors are taken along the axis of analyzer $\left(A_{\text {ap1 }}\right.$ and $\mathrm{A}_{\mathrm{ap} 2}$ ), which are out of phase according to the orientation of principal planes. Thus the resultant vector is smaller changing the intensity of the emerging light. Angles $\alpha$ and $\theta$ govern the sensitivity and dynamic range of the sensor.

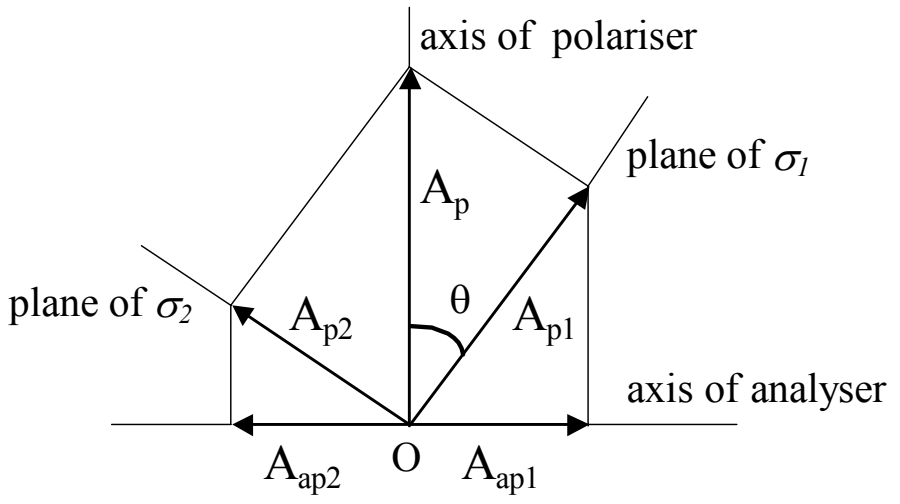

Figure 6. Vector representation of light in crossed polarizers
Equation 9 suggests that change in intensity of light at the receiver may be obtained only if there is a change in the value of $\sigma_{l}, \sigma_{2}$, other parameters being constant. Considering the slip of an object, the system of stresses is changed at the very start of the slip due to sudden energy imparted to the photoelastic material and so is the case in reverse order when the slip is terminated. But during the slip with constant rate, the stresses remained constant with pressure distribution changing due to the microscopic surface irregularity (this may not be the case when the object surface is rough). Thus for slip at constant rate, no slip signal is available. However, the sensor could provide continuous slip signal if the slip motion can partially be converted into transverse motions to change the system of stresses. The key to achieve this is to improve the surface characteristics of the slip-surface of the photoelastic layer so that it can set up a system of vibration whenever object slips over it.

\section{SENSOR CONSTRUCTION}

In an attempt to produce continuous slip signals during slippage the top surface of the photoelastic layer, which forms the slip surface, has been investigated with different geometry and material as shown in Fig. 7, and tested experimentally. It has been observed that the sensor did not provide continuous slip signals, since the projecting edges of the sensor in each case stuck to the slipping object at a constant slip rate.
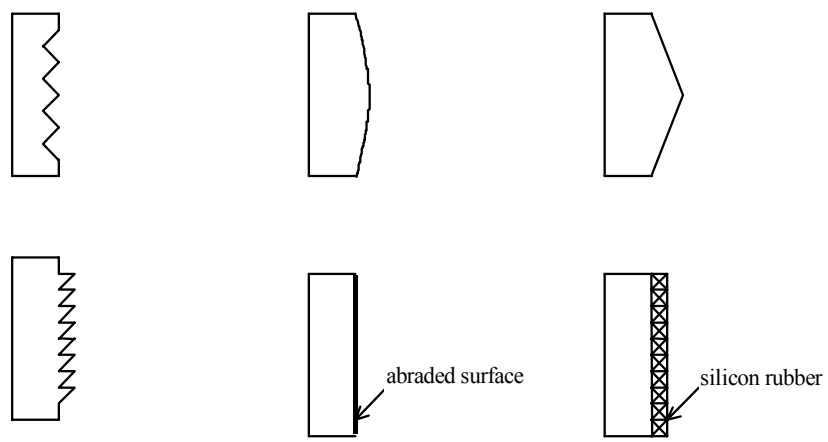

Figure 7. The non-enhancing surface changes

If sensor's edges are partially loaded by the slipping object and are just making contact with the object during slip, the vibration could be generated as reported in [20]. However, in the present study the object size was bigger than the sensor size which covers the entire surface area of the sensor, thus the sensor produced slip signals only during start and termination of the object slip. Even abrading the slip surface and changing the top surface with silicon rubber were found to be unworkable. However, when the top surface was replaced by a metallic surface, it has been found that the sensor produced a continuous slip signal when the object slipped over it and the slip signal is found to be a function of the slip rate. This behavior was exhibited because of the self-excited vibration induced between the contacting surfaces.

The phenomena of self-excited vibration due to dry friction have been observed when one object surface slides over the other. The process is caused by the fact that the frictional force does not remain constant as a function of velocity. It 
decreases as the relative velocity increases, giving rise to a negative damping to sustain the vibration of the system [24]. When the object surfaces are in contact and stationary, the relative velocity between the point of contact and the sliding surface is zero. When one surface starts sliding, a relative velocity is set and the frictional force acting at the interface displaces the point of contact which changes the relative velocity, which in turn changes the frictional force. When the relative velocity of a point of contact increases (i.e. when the second object moves against the sliding object) the magnitude of frictional force is reduced, as a result the amplitude of displacement decreases and so is the relative velocity of point of contact. This increases the magnitude of frictional force and eventually increase the amplitude of displacement and the whole cycle repeats itself setting the system to vibrate. For such a vibration to take place, the relative velocity must not cease, also the characteristic of vibration so exhibited is very much dependent on the material of the contacting surfaces which governs the change in frictional force with change in relative velocity. A complete theoretical analysis of the sensor based on frictional characteristics of different materials is available in [25], where it has been shown how vibration can be sustained and how the sensor can provide continuous slip signals at a constant slip rate.

The enhanced sensor is shown in Fig. 8 where a thin metallic strip is attached to the photoelastic layer. The metallic piece of the same surface area was glued onto the photoelastic layer (which forms the slipping surface) in such a way that it did not pre-stress the photoelastic material and with the same care the photoelastic layer was glued to a U-shaped sensor body (not shown). Care was also taken to place the polarizer/analyzer sheets in the crossed mode so that it did not interfere with the photoelastic layer. The optical fibers were fixed to the two arms of the U-shaped sensor-body on two close-fit through holes. The arrow on the sensing area (Fig. 8) shows the direction of object slip and should be kept perpendicular to the optical fiber alignment to have the photoelastic effect [26]. The light source used is a high radiance emitter of peak spectral output of 850 $\mathrm{nm}$ and the receiver is a PIN photodiode to match the emitter. The single stage detection circuit used in this experiment is shown in Fig. 9.

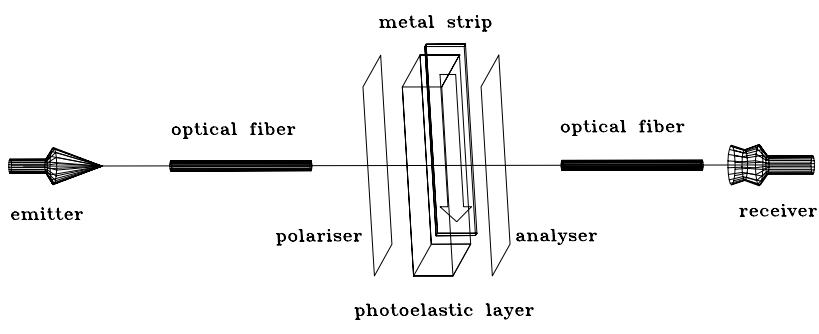

Figure 8. The enhanced sensor

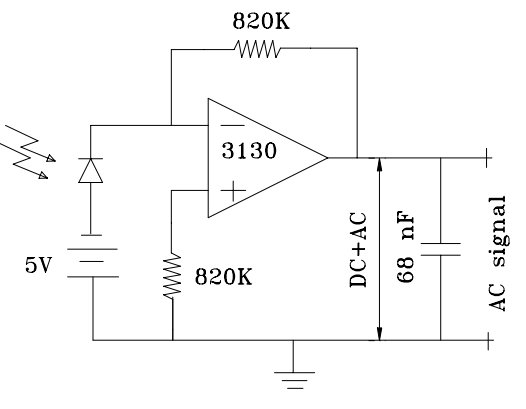

Figure 9. Detection circuit

The output from the amplifier contains both the normal force as well as the slip signals. When the signal is filtered, an AC signal is available which gives measure of slip and when the slip signal is subtracted from the main signal a DC signal is available (this has been achieved digitally in the software). This provides absolute information on the normal force. In the following experimental plots (Figs. 11-13), the upper signal is shown as the normal force and a small signal about the mean position (reading 0 ) is shown as the slip signal. The size of the sensing element was $9 \mathrm{~mm} \times 5 \mathrm{~mm} \times 3 \mathrm{~mm}$, and the sensing area is $9 \mathrm{~mm} \times 3 \mathrm{~mm}$ with a stainless steel plate of $0.3 \mathrm{~mm}$ thickness on the top. The test-rig used in this experiment is a three link articulated finger [27] as shown in Fig. 10. A small DC servomotor controls the slip of the object in vertical direction under gravity.

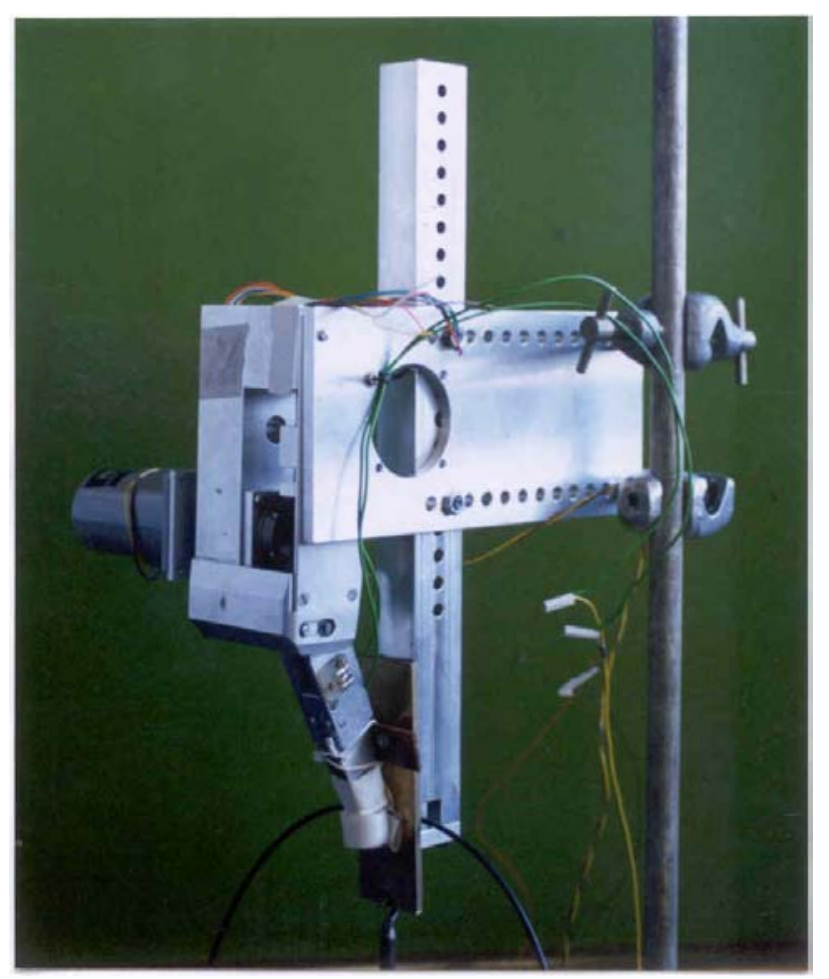

Figure 10. The test-rig 


\section{EXPERIMENTAL OBSERVATIONS}

Experiments have been conducted with the sensor to compare the slip signals for objects of different mass and material. Objects used in the test were a well machined aluminum block of average surface roughness $0.8 \mu \mathrm{m}$ and a plastic block of average surface roughness $0.5 \mu \mathrm{m}$. These objects do not have a surface roughness which can be exploited for the detection of slip. The objects are allowed to slip over the sensor surface, under gravity and the applied force from the fingertip. The finger contact surface has a rubber pad to provide a better closed-loop control of the object slip [28].

Figure 11 (a) shows the initial condition of signals as seen on an oscilloscope when no object is held. The upper plot shows the DC (normal force) signal while the lower is the AC (slip) signal in a time frame of $50 \mathrm{~ms} / \mathrm{div}$. In order to see the smaller slip rates, the AC signal has been set at $20 \mathrm{mV} / \mathrm{div}$ while due to the initial offset of the DC signal, it has been set at $2 \mathrm{~V} /$ div. Since the DC signal has been set at a high voltage, small change in the signal cannot be seen in the plot.

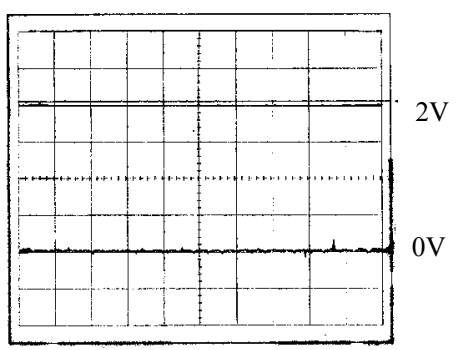

(a)

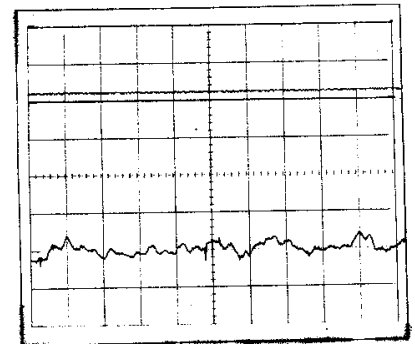

(b)
Figure 11. Initial conditions of signals, and the slip signals

Figure 11 (b) shows the change in signals when a plastic block of $155 \mathrm{~g}$ is slipping. Due to the applied force, the DC signal is seen to increase from the previous value and the $\mathrm{AC}$ signal is seen to be perturbed. The variation exists throughout the time-range indicating that the slip signal is available continuously. The object slip rate in this case was $2.7 \mathrm{~mm} / \mathrm{s}$. When the same object is allowed to slip at a lower rate $(0.72$ $\mathrm{mm} / \mathrm{s}$ ), the amplitude as well as the frequency of the signal is seen to decrease, Fig. 12(a). The same experiment was repeated with an aluminum block of $105 \mathrm{~g}$ slipping at a rate of 0.61 $\mathrm{mm} / \mathrm{s}$, Fig. 12(b). Other conditions being the same, it can be seen that for approximately the same slip rate as in Fig. 12(a) (for the plastic block), the amplitude of the slip signal is higher for the metallic object as compared to the plastic object. It can be concluded that the slip signal has a high amplitude with metallic objects than that with non-metallic objects at the same slip rate, since the friction-velocity relations are different for different materials.

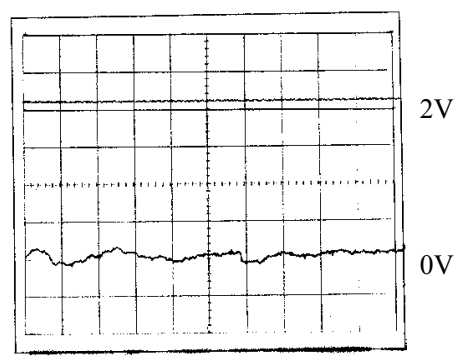

(a)

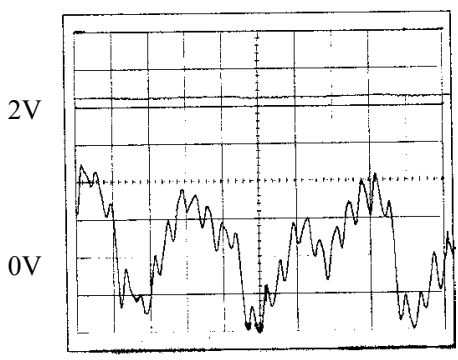

(b)
Figure 12. Slip signal for different materials at same slip rate

Figure 13(a) shows the signals obtained when the aluminum block slips at $3 \mathrm{~mm} / \mathrm{s}$. It can be seen that the amplitude, in this case is very high which also affects the applied force signal (in the upper plot), since the signal has both AC and DC components. The slip signal is seen to be irregular and the frequency of the signal is found to be higher than the previous case in Fig. 12(b), means that for the same material at different slip rates the sensor provides different signals. The sensor is found to be capable of detecting slip rates as small as $0.1 \mathrm{~mm} / \mathrm{s}$ for certain metallic objects as can be seen from Fig. 13(b). In all the above cases a change in DC signal provides the normal force information. Due to high initial offset, the DC signal cannot be easily differentiated in these plots for different forces applied to the object. However, the sensor has been calibrated for the normal force to fully characterize it. Further, the sensor has been tested against external disturbances by imparting artificial jerks and shocks to the test rig. It has been found that the sensor is largely immune to such disturbances and the signals remain spike-free.

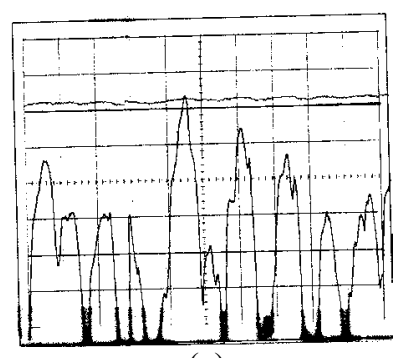

(a)

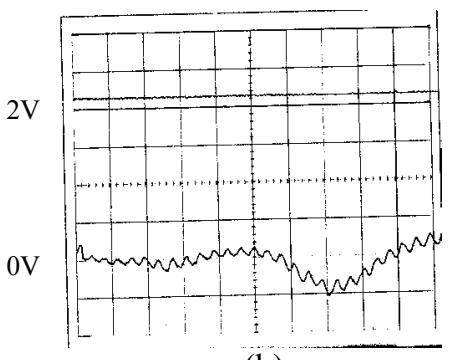

(b)
Figure 13. Slip signal for same materials at different slip rates

\section{SENSOR CALIBRATION FOR NORMAL FORCE}

The sensor was calibrated for the normal force. The plot of applied force against voltage (which is proportional to the light intensity at the receiver) is shown in Fig. 14. It can be seen that the variation closely follows the sine-squared function as has been predicted theoretically [23]. For the present sensor, the voltage increases up to a force of $1.7 \mathrm{~N}$, after that the voltage drops to the initial condition providing a total force range of 6 $\mathrm{N}$. The sensor can be used until $1.7 \mathrm{~N}$ for absolute applied force measurement, however, the full range of $6 \mathrm{~N}$ can be exploited by suitably processing the signal to track the increasing or the decreasing trend of the voltage. 


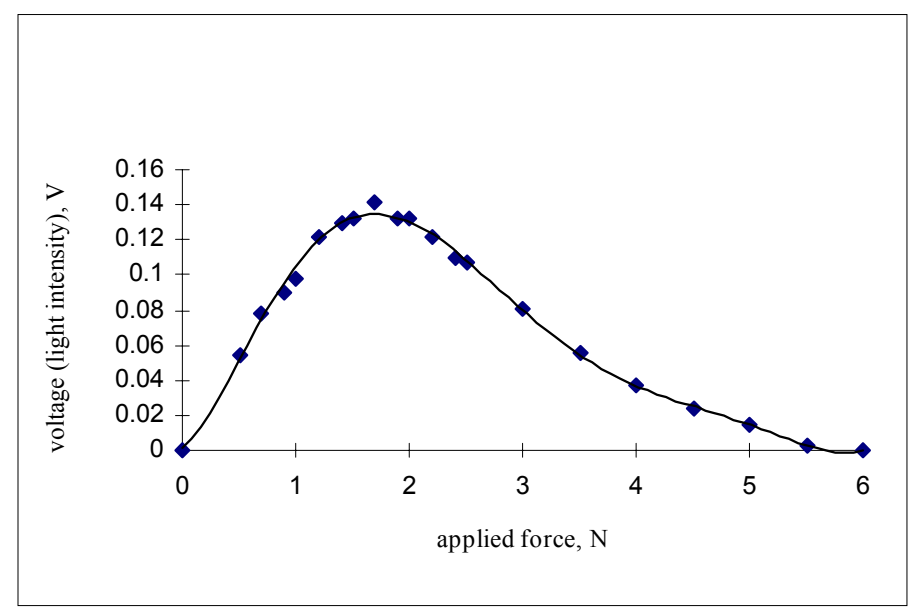

Figure 14. Sensor calibration graph for the normal force

The limitation of the sensor, as is true in case of any other pressure sensors, has been experienced due to contact localization of the applied force. Since the sensor works on the stress developed in the photoelastic material, the reading could be misleading if the contact is localized at one end and is detected at the centre or at the other end of the sensor. In order to improve the sensor on this account, more optical input/output fibers can be used throughout the length of the sensor and the resulting signal should be averaged-out and calibrated accordingly. The range of the applied force can be improved by increasing the contact area of the sensor, however, for many applications area enlargement may not be allowed. The other possibility is to change the photoelastic material from polyurethane to acrylic or epoxy to provide higher force range [29]. Further for compactness, the optical fibers can be discarded and U-shaped opto-switch with emitter detector of matching wavelength could be used.

\section{DISCUSSION AND CONCLUSIONS}

In this paper mechanism of slip has been investigated to develop a sensor for providing continuous signal during slip. The paper presented theoretical aspect as well as the experimental results of the developed sensor. The theoretical model helps identify various sensor parameters involved in the sensor design for the resulting signal. The sensor is novel in the sense that it provides information on slip as well as normal force with a significant improvement of delivering continuous signal during slip. Such sensors could be advantageous for grasping and manipulation operation since they can extract slip and applied force information from a single contact location. The sensor is found to be capable of detecting fine scratches available on the object surface, which can be exploited for quality assessment of object surfaces. Besides this, the overall size of the sensor is so small that it can be easily incorporated into a robotic finger. The top metallic surface of the sensor is wear resistant which can be an added advantage from an application point of view. It offers immunity to the external mechanical vibrations which has the potential for use in robotics and prosthetic applications.
As the sensor works on the change in the coefficient of friction between the sensor surface and the slipping object, the slip signal in case of some materials may be more pronounced than in others. Particularly the metallic objects are expected to provide stronger signals as compared to the non-metallic objects since the variation of the coefficient of friction due to sliding speed is higher in metal-metal contacts as compared to that in metal-nonmetal combinations.

While attempting to improve the sensor compactness, the sensor design was changed to incorporate the emitter and the detector directly into a U-slot with the photoelastic layer. The new design was however found to be unworkable. This was due to the available size of emitter and detector which had a larger diameter $(6-7 \mathrm{~mm})$ as compared to the optical fibers diameter (2 mm). When these optoelectronic devices were placed directly in the U-slot, the center of signal detection was shifted away from the top surface. Since slip is a surface phenomenon, the effect of object slip far away from the surface was very much diminished. As a result, the stress change in photoelastic material due to vibration was not received at the detector. The application however, would definitely improve if the emitter detector of small size (1-2 mm) can be directly placed closed to the slipping surface.

From the analysis presented, it is apparent that the size of sensor is an important consideration in slip sensing. Bigger the size of the sensor with respect to the object size, it can provide slip signal as the object moves over the contact area but in case of bigger object size over a smaller sensing area, auxiliary effect of slippage need to be used to get continuous slip signal as discussed in this paper. From the theoretical analysis of the sensor, it can be concluded that an accurate model of the sensor based on self-excited vibration is very difficult to develop. Such a system cannot be modeled precisely since many parameters are involved which cannot be determined independently. The analysis, however, helps to identify various sensor parameters which affect the resulting signal from the sensor. Since the sensor works on vibration of the sensing medium with a very small amplitude, construction of the sensor should provide a room for the elastic freedom of the photoelastic material and should not have large damping. The above discussion suggests that a large number of parameters are involved for the resulting signal. The behavior of the sensor also depends on the method of construction employed. The fixing of photoelastic material with the polarizer and analyzer plays a major role. Excessive gluing of these components in the sensor body may pre-stress the photoelastic material lowering the sensitivity and the dynamic range of the sensor. The placement of the metallic strip over the sensor surface and the optical fiber connections also requires careful considerations. Besides this, the signal from the sensor also depends on the way it interacts with the sliding object, for example the type of the motion the slipping object has (rotational, translational or a combination of these). All these factors are very much involved in the operation of the sensor for the resulting signal. However, the presented design is simple and yet robust as compared to the other existing designs. 
We are currently researching on creating a photoelastic fingertip with integrated emitter and detector and in-built polarizer and analyzer to investigate how the system parameters affect the sensor behavior. Even though the sensor provides different signals for different materials and slip rates, the signals from the sensor can be intelligently processed [30] to obtain useful information for grasping and manipulation tasks. It is our belief that an integrated system will be viable for a number of applications in robotics and prosthetics.

\section{ACKNOWLEDGMENTS}

The Faculty of Engineering \& Applied Science, Department of Electrical Engineering of University of Southampton, and the Overseas Research Student award from the UK Committee of Vice-Chancellors and Principals have supported this research.

\section{REFERENCES}

[1] Yoshikawa, T., Yokokohji, Y. and Nagayama, A., 1993 "Object handling by three-fingered hands using slip motion" Proc. of the IEEE/RSJ Intl. Conf. on Intelligent Robots and Systems, pp. 99-105

[2] Todd, R. W. and Nightingale, J. M., 1969 "Adaptive prehension control for a prosthetic hand" 3rd Intl. Sympos. on external control of human extremities.

[3] Howe, R. D., 1994 "Tactile sensor for localizing transient events in manipulation" Proc. of the IEEE intl. Conf. on Robotics and Automation. pp. 471-476.

[4] Cutkosky, M. R., and Hyde, J. M., 1993 "Manipulation control with dynamic tactile sensing" Proc. of the 6th ISRR, Pennsylvania.

[5] Magnussen, B. and Doersam, T., 1995 "The Karlsruhe dextrous hand, a three fingered robot gripper" Proc. of the European control conference, Rome.

[6] Crowder, R. M., 1988 "Sensors, touch, force and torque measurement" R. C. Dorf edited International Encyclopaedia of Robotics, John Wiley \& Sons, New York, pp. 1544-1563.

[7] Klafter, R. D., Chmielewski, T. A. and Negin, M., 1989 "Robotic Engineering : An integrated approach" Prentice -Hall International, Inc.

[8] Dominguez-Lopez, J. A., Damper, R. I., Crowder, R. M. and Harris, C. J., 2004 "Adaptive neurofuzzy control of a robotic gripper with online machine learning" Robotics and Autonomous Systems 48(2-3):pp. 93-110.

[9] Kyberd, P. J. and Chappell, P. H., 1992 "Characterization of an optical and acoustic touch and slip sensor for autonomous manipulation" Measurement Science \& Technology, Vol. 3, No. 10, pp. 969-975.
[10] Kvasnik, F., Jones, B. E. and Beck, M. S., 1985 "Photoelastic slip sensors with optical fiber links for use in robotic grippers" Institute of Physics Conf. on sensors, Southampton, UK.

[11] Eghtedari F. and Morgan C., 1989 "A novel tactile sensor for robot applications” Robotica Vol. 7, pp. 289-295.

[12] Nishihara, K., Komiya, S., Okuma, N. and Otsuka, H., 1996 "Slip sensor using a piezoelectric bimorph element" Trans. of the JSME, part C, Vol. 62, No. 598, pp. 2244-2249.

[13] Holweg, E. G. M., Hoeve, H., Jongkind, W., Marconi, L., Melchiorri, C. and Bonivento, C., 1996 "Slip detection by tactile sensors: algorithm and experimental results" Proc. IEEE Intl. Conf. on Robotics and Automation, pp. 3234-3239.

[14] Cotton, D., Cranny, A., White, N., Beeby, S. and Chappell, P., 2004 "Design and development of intergrated thick-film sensors for prosthetic hands" Proc. of the 7th Biennial Conference on Engineering Systems Design and Analysis, v 3, pp 573-589.

[15] Kyung, K., Son, S., Kwon, Dand Kim, M. 2004 "Design of an integrated tactile display system" Proc. of the IEEE International Conference on Robotics and Automation, vol 1, pp. 776-781.

[16] Engel, J., Chen, J., Liu, C., Flachsbart, B., Selby, J., and Shannon, M. 2002, "Development of polyimide-based flexible tactile sensing skin Materials" Research Society Symposium Proceedings, v 736, pp. 165-170.

[17] Yamada, Y., Fujimoto, I., Morizono, T., Umetani, Y., Maeno, T.and Yamada, D., 2001 "Development of artificial skin surface ridges with vibrotactile sensing elements for incipient slip detection" IEEE International Conference on Multisensor Fusion and Integration for Intelligent Systems, pp. 251-257.

[18] Sasaki, K., Hirota, T., Fujikake, Y. and Nakaki, H., 2001 "Signal processing for slip and contact sensing and its application to a two-fingered robotic hand" Integrated Computer-Aided Engineering, v8, n4, pp. 283-291.

[19] De Rossi, D., 1991 "Artificial tactile sensing and haptic perception" Measurement, Science and Technology, Vol. 2, pp. 1003-1016.

[20] Tremblay, M. R., Packard, W. J., and Cutkosky, M. R. [1992] "Utilising sensed incipient slip signals for grasp force control" Proc. Japan-USA Symp. on flexible automation, Part 1, pp. 1237-1243.

[21] Tremblay, M. R., and Cutkosky, M. R. [1993] "Estimating friction using incipient slip sensing during a manipulation task" Proc. of the IEEE intl. Conf. on Robotics and Automation. pp. 429-434. 
[22] Timoshenko, S. P. and Gere, J. M., 1972 "Mechanics of materials" Van Nostrand Reinhold Company.

[23] Heywood R. B., 1969 "Photoelasticity for Designers" Pergamon Press, London.

[24] Rabinowicz, E., 1965 "Friction and wear of materials" John Wiley and Sons, Inc.

[25] Dubey, V. N., 1997, "Sensing and control within a robotic end effector", PhD Thesis, University of Southampton, UK.

[26] Eghtedari, F., Hopkins, S. H. and Pham, D. T., 1993 "Model of a slip sensor", Proc. of Inst. of Mech. Engrs. part B, Vol. 207, pp. 55-64.

[27] Dubey, V. N. and Crowder, R. M., 2004 "Grasping and control issues in adaptive end effectors" ASME International Design Engineering Technical Conference, DETC2004-57126, Salt Lake City, Utah (USA), Sept. 28-Oct. 2.

[28] Crowder, R.M., Dubey, V.N. and Chappell, P.H., 2000, "Dynamic Control of fingertip force using Tactile Sensors and Fuzzy Logic", Sixth International Conference on Control, Automation, Robotics and Vision, Singapore.

[29] Jacobsen, S. C., Wood, J. E., Knutti, D. F. and Biggers, K. B., 1986 "The Utah/MIT dextrous hand: work in progress" in D.T. Pham and W.B. Heginbotham edited Robot Grippers, pp 341-389.

[30] Dubey, V.N., Crowder, R.M. and Chappell, P.H., 1999, "Optimal object grasp using tactile sensors and fuzzy logic" Robotica, Vol. 17, No. 6, pp. 685-693. 